\title{
Levofloxacin treatment does not prevent BK virus infection
}

A 3-month course of levofloxacin does not prevent BK viruria after kidney transplantation but does increase the risk of adverse events, according to data from a new study. "Quinolone prophylaxis was ineffective in preventing BK viruria ... this finding contradicts previous observational data," says senior author John Gill.

The BK virus lies dormant in $60-80 \%$ of the general population, and suppression of the immune system following kidney transplantation can allow it to reactivate. Infection can ultimately lead to BK virus nephropathy, for which no effective treatment exists. "The current reactive approach to BK virus managementdecreasing immunosuppression in response to infection-remains inadequate," explains Gill. "We therefore tested whether an approach of prophylaxis could prevent infection."

Previous observations from retrospective studies indicated that quinolone antibiotics can reduce the incidence of BK viruria and viraemia -the initial signs of infection-in patients who have received kidney transplants. Whereas these observations were based on retrospective studies, Gill and colleagues tested the effectiveness of one such antibiotic in a double-blind, randomized controlled trial.

\section{4 ...levofloxacin is ineffective} in preventing BK infection ... so should not be used for this purpose 77

In this study, 154 kidney transplant recipients were randomly assigned to receive either levofloxacin or placebo. Treatment started within 5 days of transplantation and continued for 3 months. Over a follow-up period of up to 1 year, patients were assessed for signs of BK infection; the primary end point was the development of BK viruria.

Neither the incidence nor the time to development of BK viruria differed significantly between the two groups. Secondary outcomes, including BK viraemia, blood and urine viral load and acute rejection, were also comparable between the groups, as was renal function throughout the duration of the study.

The overall incidence of infections was similar between the groups; however, patients on levofloxacin had an increased risk of resistant infection with isolates usually sensitive to quinolones. A nonsignificant increase in the risk of tendonitis was also seen among patients on levofloxacin. "This study provides strong evidence that prophylactic use of levofloxacin is ineffective in preventing BK infection and is associated with increased adverse events so should not be used for this purpose," concludes Gill.

Ian Fyfe

Original article Knoll, G. A. et al. Levofloxacin for BK virus prophylaxis following kidney transplantation: a randomized clinical trial. JAMA doi:10.1001/jama.2014.14721 